\title{
Editorial for the SoSyM issue 2015/03
}

\author{
Jeff Gray $^{1}$ - Bernhard Rumpe ${ }^{2}$
}

Published online: 17 June 2015

(C) Springer-Verlag Berlin Heidelberg 2015

\section{New structure and organization of the editorial-board}

During the illness and recent loss of our dear colleague, Robert France, additions were made to the SoSyM editorial team to assist with the responsibilities associated with the review assignment and notification of submitted manuscripts. The team now welcomes Jeff Gray, who served previously as editor since 2008 and was Guest Editor of four special issues. The SoSyM organization is now structured as follows:

- Jeff Gray, Editor-in-Chief; responsible for regular papers

- Bernhard Rumpe, Editor-in-Chief; responsible for special and theme sections, and expert voices

- Marsha Chechik, Martin Gogolla, Jean-Marc Jezequel, Associate Editors; responsible for managing the review process for regular papers

- Geri Georg, Assistant Editor; regular papers

- Martin Schindler, Assistant Editor; special and theme sections, expert voices, and finalizing each issue

By way of introduction, Jeff Gray is a Professor in the Department of Computer Science at the University of Alabama (UA). Jeff received a Ph.D. in Computer Science from the Department of Electrical Engineering and Computer Science at Vanderbilt University. As a research assistant at the Institute for Software Integrated Systems, Jeff's mentors were

$\triangle$ Bernhard Rumpe

bernhard.rumpe@sosym.org

Jeff Gray

jeff.gray@sosym.org

1 University of Alabama, Tuscaloosa, AL, USA

2 RWTH Aachen University, Aachen, Germany
Gabor Karsai and Janos Sztipanovits. He also received a BS and MS degree in Computer Science from West Virginia University. Jeff is a National Science Foundation CAREER award recipient and was named the 2008 Carnegie Foundation Professor of the Year. Jeff is a Distinguished Member of the ACM and a Senior Member of the IEEE, and he currently serves as the chair of the Alabama IEEE Computer Society. He also serves on the Education Advisory Council of Code.org.

Jeff was the co-Chair of MODELS 2013 and the Organizing Chair of AOSD 2009, among several PC Chair assignments (over 70 organizing and 205 program committees). He was co-founder of the ICMT conference and the long-running Domain-Specific Modeling workshop at OOPSLA/SPLASH. His research has been funded by NSF, Google, DARPA, IBM, US Air Force, and US Department of Education. In addition to research in software modeling, Jeff has worked for over a decade on Computer Science Education for young children, offering summer camps, various competitions, and teacher training. More about Jeff's work is available at: http://gray.cs.ua.edu/.

SoSyM continues to have a large and distinguished Editorial Board, consisting of contributing members who are active and well-known in the modeling community. The Editorial Board is balanced in many ways - across areas of software modeling research topic areas, as well as geography (although Asia is still underrepresented). The current membership period of the SoSyM Editorial Board does not have term limits and continues to have a moderate workload, despite a growing number of submissions.

With our new team, we are confident that SoSyM can further serve as an outstanding venue for publications about modeling, modeling languages, use of models in software and systems development and related topics. The scope of SoSyM also extends beyond Computer Science and Engineering to 
also cover interests in the natural and social science communities, when dealing with complex software and systems models.

\section{Future SoSyM page budget}

The Impact Factor of a journal is only calculated for papers that have been cited over the past 2 years. Our experience suggests that journal papers are cited more often when they reach their final form in a printed publication. To help reduce the backlog of the SoSyM publication pipeline and to improve the opportunity for citation, we have arranged through Springer to produce additional pages in each SoSyM issue. Springer has been printing SoSyM at an enlarged size since 2013. They have agreed to increase the page budget for each issue from 256 to 294 pages, which means 1,176 pages overall will be available each year.

\subsection{SoSyM is soliciting survey articles}

In order to improve SoSyM by addressing community needs, we asked readers and authors to suggest the topics and themes that they would like to see in future issues. Several responses suggested the need for survey/overview articles that address specific areas of software and systems modeling. Therefore, we encourage the submission of survey articles that are of excellent quality (e.g., a systematic literature review of a SoSyM topic area, or a deep overview of a specific area that summarizes past work and synthesizes a future research plan while also identifying open problems).

If you have an idea for a survey paper about a modeling theme, please consider submitting a scientific assessment in the form of an article to SoSyM. Survey articles will still be reviewed according to high-quality standards, but do not need to contain a research experiment that has novel findings. Authors may also contact us before submission and provide a suggested topic area, list of authors, and a short argument that motivates the importance of the area surveyed. Other items that could be included in the correspondence are: why are the proposed authors the appropriate experts to serve as an authority to write such a survey, what comparison and evaluation criteria (e.g., epistemological, empirical, or any analytical form) will be used to categorize or distinguish the contributions made in the area. An outline, as well as an initial list of surveyed papers, tools, or approaches may be included in the correspondence.

\subsection{Content of this issue}

This issue includes a special section on artifacts and processes for business process modeling and management (BPMDS). The special section was organized by Selmin Nurcan and Rainer Schmidt and contains five papers. The guest editorial by Selmin and Rainer is included as a preface to the special section. This issue also contains the following five regular papers. ${ }^{1}$

- Localized Model Transformations for Building LargeScale Transformations by Anne Etien, Alexis Muller, Thomas Legrand, and Richard Paige

- T-Core: A Framework for Custom-built Model Transformation Engines by Eugene Syriani, Hans Vangheluwe, and Brian LaShomb

- A Demonstration-based Model Transformation Approach to Automate Model Scalability by Yu Sun, Jeff Gray ${ }^{1}$, and Jules White

- An Interactive Tool for UML Class Model Evolution in Database Applications by Dragan Milicev and Vukasin Milovanovic

- Converting Metamodels to Graph Grammars: Doing Without Advanced Graph Grammar Features by Luka Fürst, Marjan Mernik, and Viljan Mahnic

We hope that this issue of SoSyM continues to inspire your current and future research!

\footnotetext{
${ }^{1}$ Coincidentally, this issue also includes a paper by Jeff Gray and coauthors that was submitted, reviewed, and published online over the past two years. It is the SoSyM policy that Editors and Associate Editors are encouraged to publish in SoSyM, but the Editors-in-Chiefs are not permitted to submit papers for review during their time of service.

The review and online publication occurred well before Jeff was considered for his new role with SoSyM.
} 